\title{
Multi-scale event synchronization analysis for unravelling climate processes: a wavelet-based approach
}

\author{
Ankit Agarwal ${ }^{1,2,3}$, Norbert Marwan ${ }^{2}$, Maheswaran Rathinasamy ${ }^{4}$, Bruno Merz ${ }^{1,3}$, and Jürgen Kurths ${ }^{1,2,5}$ \\ ${ }^{1}$ University of Potsdam, Institute of Earth and Environmental Science, Karl-Liebknecht-Strasse 24-25, \\ 14476 Potsdam, Germany \\ ${ }^{2}$ Potsdam Institute for Climate Impact Research, P.O. Box 6012 03, 14412 Potsdam, Germany \\ ${ }^{3}$ GFZ German Research Centre for Geosciences, Section 5.4: Hydrology, Telegrafenberg, Potsdam, Germany \\ ${ }^{4}$ Civil engineering department, MVGR college of Engineering, Vizianagaram, India \\ ${ }^{5}$ Institute of Applied Physics of the Russian Academy of Sciences, 46 Ulyanova St., Nizhny Novgorod 603950, Russia
}

Correspondence to: Ankit Agarwal (aagarwal@uni-potsdam.de)

Received: 3 May 2017 - Discussion started: 12 June 2017

Revised: 1 September 2017 - Accepted: 9 September 2017 - Published: 13 October 2017

\begin{abstract}
The temporal dynamics of climate processes are spread across different timescales and, as such, the study of these processes at only one selected timescale might not reveal the complete mechanisms and interactions within and between the (sub-)processes. To capture the non-linear interactions between climatic events, the method of event synchronization has found increasing attention recently. The main drawback with the present estimation of event synchronization is its restriction to analysing the time series at one reference timescale only. The study of event synchronization at multiple scales would be of great interest to comprehend the dynamics of the investigated climate processes. In this paper, the wavelet-based multi-scale event synchronization (MSES) method is proposed by combining the wavelet transform and event synchronization. Wavelets are used extensively to comprehend multi-scale processes and the dynamics of processes across various timescales. The proposed method allows the study of spatio-temporal patterns across different timescales. The method is tested on synthetic and real-world time series in order to check its replicability and applicability. The results indicate that MSES is able to capture relationships that exist between processes at different timescales.
\end{abstract}

\section{Introduction}

Synchronization is a widespread phenomenon that can be observed in numerous climate-related processes, such as synchronized climate changes in the northern and southern polar regions (Rial, 2012), see-saw relationships between monsoon systems (Eroglu et al., 2016), or coherent fluctuations in flood activity across regions (Schmocker-Fackel and Naef, 2010) and among El Niño and the Indian summer monsoon (Maraun and Kurths, 2005; Mokhov et al., 2011). Synchronous occurrences of climate-related events can be of great societal relevance. The occurrence of strong precipitation or extreme runoff, for instance, at many locations within a short time period may overtax the disaster management capabilities.

Various methods for studying synchronization are available, based on recurrences (Marwan et al., 2007; Donner et al., 2010; Arnhold et al., 1999; Le Van Quyen et al., 1999; Quiroga et al., 2000, 2002; Schiff et al., 1996), phase differences (Schiff et al., 1996; Rosenblum et al., 1997), or the quasi-simultaneous appearance of events (Tass et al., 1998; Stolbova et al., 2014; Malik et al., 2012; Rheinwalt et al., 2016). For the latter, the method of event synchronization (ES) has received popularity owing to its simplicity, in particular within the fields of brain (Pfurtscheller and Silva 1999; Krause et al., 1996) and cardiovascular research (O'Connor et al., 2013), non-linear chaotic systems (Callahan et al., 1990), and climate sciences (Tass et al., 1998; Stolbova et al., 2014; Malik et al., 2012; Rheinwalt et al., 2016). ES 
has also been used to understand driver-response relationships, i.e. which process leads and possibly triggers another based on its asymmetric property. It has been shown that, for event-like data, ES delivers more robust results compared to classical measures such as correlation or coherence functions which are limited by the assumption of linearity (Liang et al., 2016).

Particularly in climate sciences, ES has been successfully applied to capture driver-response relationships, time delays between spatially distributed processes, strength of synchronization, and moisture source and rainfall propagation trajectories, and to determine typical spatio-temporal patterns in monsoon systems (Stolbova et al., 2014; Malik et al., 2012; Rheinwalt et al., 2016). Furthermore, extensions of the ES approach have been suggested to increase its robustness with respect to boundary effects (Stolbova et al., 2014; Malik et al., 2012) and number of events (Rheinwalt et al., 2016).

Even though ES has been successfully used, it is still limited by measuring the strength of the non-linear relationship at only one given temporal scale, i.e. it does not consider relationships at and between different temporal scales. However, climate-related processes typically show variability at a range of scales. Synchronization and interaction can occur at different temporal scales, as localized features, and can even change with time (Rathinasamy et al., 2014; Herlau et al., 2012; Steinhaeuser et al., 2012; Tsui et al., 2015). Features at a certain timescale might be hidden while examining the process at a different scale. Also, some of the natural processes are complex due to the presence of scale-emergent phenomena triggered by non-linear dynamical generating processes and long-range spatial and long-memory temporal relationships (Barrat et al., 2008). In addition, single-scale measures, such as correlation and ES, are valid and meaningful only for stationary systems. For non-stationary systems, they may underestimate or overestimate the strength of the relationship (Rathinasamy et al., 2014).

The wavelet transform can potentially convert a nonstationary time series into stationary components (Rathinasamy et al., 2014), and this can help in analysing nonstationary time series using the proposed method.

Therefore, the multi-scale analysis of climatic processes holds the promise of better understanding the system dynamics that may be missed when analysing processes at one timescale only (Perra et al., 2012; Miritello et al., 2013). According to this background, we propose a novel method, multi-scale event synchronization (MSES), which integrates ES and the wavelet approach in order to analyse synchronization between event time series at multiple temporal scales. To test the effectiveness of the proposed methodology, we apply it to several synthetic and real-world test cases.

The paper is organized as follows: Sect. 2 describes the proposed methodology and Sect. 3 introduces selected case studies. The results are discussed in Sect. 4. Conclusions are summarized in Sect. 5.

\section{Methods}

Here we describe the methodology for the proposed MSES approach. In this we combine two already well-established approaches (DWT and ES) to analyse synchronization at multiple temporal scales. The following sub-sections briefly introduce wavelets and ES and subsequently provide the mathematical framework for estimating MSES.

\subsection{Discrete wavelet transform}

Wavelet analysis has become an important method in spectral analysis due to its multi-resolution and localization capability in both time and frequency domains. A wavelet transform converts a function (or signal) into another form which makes certain features of the signal more amenable to study (Addison, 2005). A wavelet $\psi(t)$ is a localized function which satisfies certain admissibility conditions. The wavelet transform $T_{a, b}(x)$ of a continuous function $x(t)$ can be defined as a simple convolution between $x(t)$ and dilated and translated versions of the mother wavelet $\psi(t)$ :

$T_{a, b}(x)=\int_{-\infty}^{\infty} x(t) \psi_{a, b}(t) \mathrm{d} t$,

where $a$ and $b$ refer to the scale and location variables (real numbers) and $\psi_{a, b}$ is defined as

$\psi_{a, b}(t)=\frac{1}{\sqrt{a}} \psi\left(\frac{t-b}{a}\right)$.

Depending on the way we sample parameters $a$ and $b$, we get either a continuous wavelet transform (CWT) or a discrete wavelet transform (DWT). A natural way to sample $a$ and $b$ is to use a logarithmic discretization of the scale and link this in turn to the size of steps taken between $b$ locations. This kind of discretization of the wavelet has the form

$\psi_{\lambda, q}(t)=\frac{1}{\sqrt{a_{0}^{\lambda}}} \psi\left(\frac{t-q b_{o} a_{o}^{\lambda}}{a_{o}^{\lambda}}\right)$

where the integers $\lambda$ and $q$ control the wavelet dilation and translation, respectively; $a_{o}$ is a specified fixed dilation step parameter and $b_{o}>0$ is the location parameter. The general choices of the discrete wavelet parameters $a_{o}$ and $b_{o}$ are 2 and 1 , respectively. This is known as dyadic grid arrangement. 


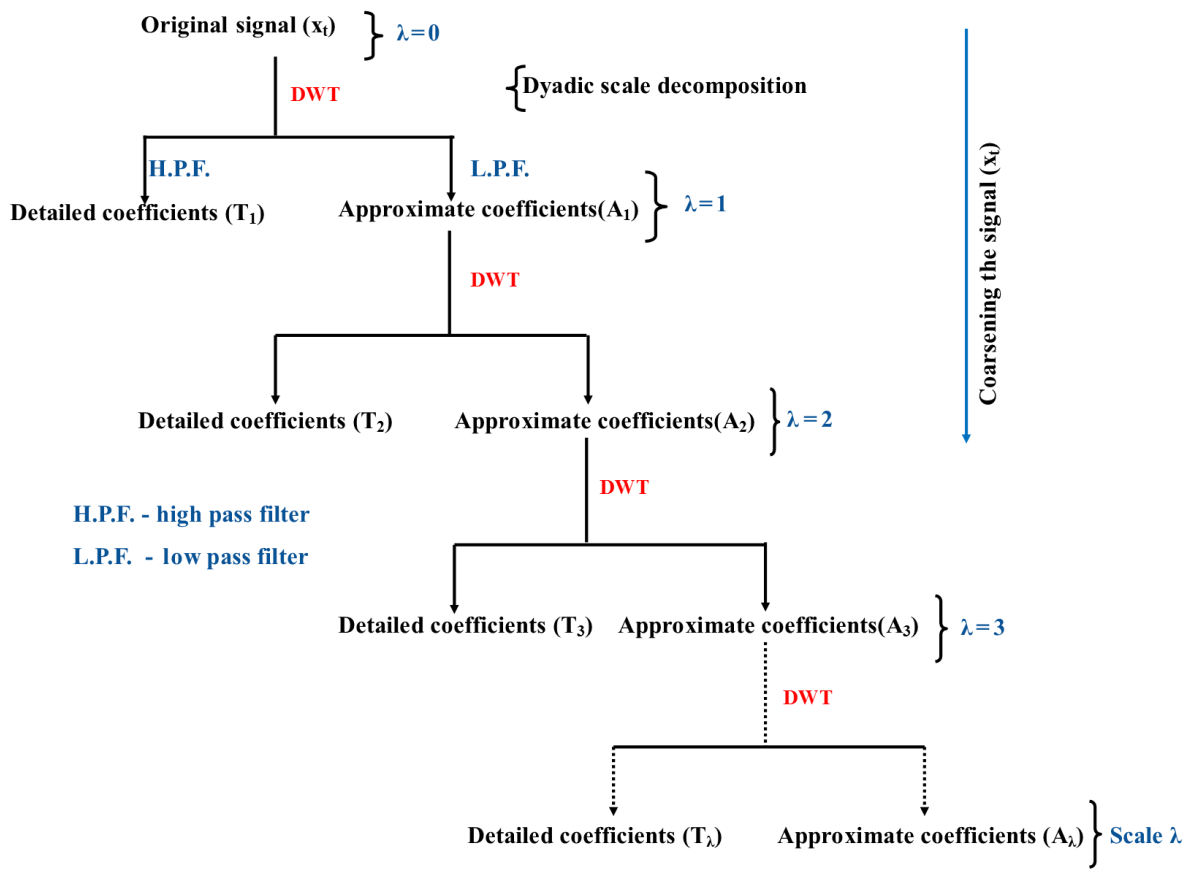

Figure 1. Schematic showing the decomposition tree for signal $X_{t}$ using DWT.

Using the dyadic grid wavelet, the DWT can be written as

$T_{\lambda, q}=\int_{-\infty}^{\infty} x(t) \frac{1}{\sqrt{a_{o}^{\lambda}}} \psi\left(\frac{t-q b_{o} a_{o}^{\lambda}}{a_{o}^{\lambda}}\right) \mathrm{d} t$. Substituting $a_{0}=2$

and $b_{o}=1$, weget

$T_{\lambda, q}=\int_{-\infty}^{\infty} x(t) 2^{-\lambda / 2} \psi\left(2^{-\lambda} t-q\right) \mathrm{d} t$

where $T_{\lambda, q}$ are the discrete wavelet transform values given on scale-location grid indexes $\lambda$ and $q$. For the DWT, the values $T_{\lambda, q}$ are known as wavelet coefficients or detail coefficients.

The decomposition of the dyadic discrete wavelet is also associated with the scaling function $\phi_{\lambda, q}(t)$ (Eq. 5) which represents the smoothing of the signal and has the same form as the wavelet, given by (Addison, 2005)

$\phi_{\lambda, q}(t)=2^{-\frac{\lambda}{2}} \phi\left(2^{-\lambda} t-q\right)$.

The scaling function is orthonormal to the translation of itself, but not to the dilation of itself. $\phi_{\lambda, q}(t)$ can be convolved with the signal to produce approximation coefficients at a given scale as follows:

$A_{\lambda, q}=\int_{-\infty}^{\infty} x(t) \phi_{\lambda, q}(t) \mathrm{d} t$.

The approximation coefficients at a specific scale $\lambda$ are known as a discrete approximation of the signal at that scale. As proven in Mallat (1989), the wavelet function and the scaling function form multi-resolution bases resulting in a pyramidal algorithm. The decomposition methodology is schematically shown in Fig. 1.

In this study, to calculate the synchronization at multiple scales, we only consider the approximation coefficients (not detail coefficients) at that particular scale because the aim is to separate the effects of time-localized features and highfrequency components from the signal.

For different $\lambda=1,2,3, \ldots$, the approximation coefficients $A_{\lambda}$ correspond to the "coarse-grained" original signal after removal of the details at scales $\lambda, \lambda-1, \ldots, 1$. In practical terms, considering a daily climatic time series at $\lambda=0$, the time series represents the original observations. At $\lambda=1, A_{1}$ represents the features beyond the 2-day scale (wavelet scale) which is obtained by extracting $T_{1}$ (2-day features) from the original time series. Similarly, at $\lambda=3, A_{3}$ represents the climatic variable beyond the 8-day scale and is obtained after removing $T_{1}, T_{2}$, and $T_{3}$ (2-, 4-, and 8-day features) from the original signal. In essence, $A_{1}, A_{2}, A_{3}, \ldots$ represent the original signal at different timescales. The schematic plot explaining the procedure and relationship between signal, approximate component, and detailed component has been shown in Fig. 2.

For simplicity we denote the approximation coefficient $A_{\lambda, q}$ of the signal $x(t)$ at scale $\lambda$ as $x_{\lambda}$. 


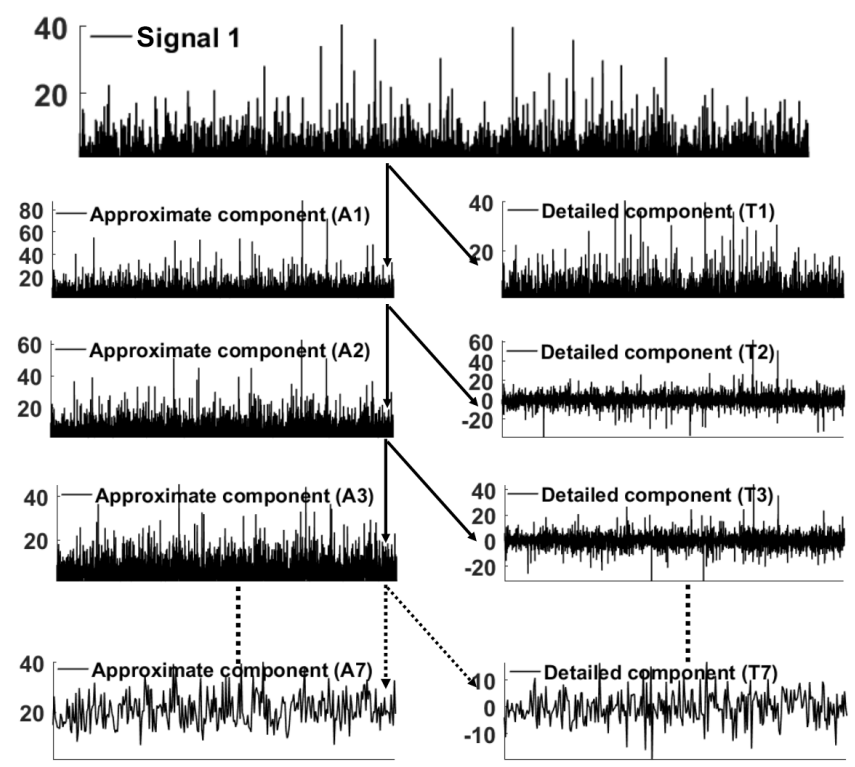

Figure 2. Scheme of multi-scale decomposition of signals using discrete wavelet transformation (DWT). The relationship between signal, approximate component, and detailed component is shown.

\subsection{Event synchronization}

To quantify the synchronous occurrence of events in different time series, we use the event synchronization (ES) method proposed by Quiroga et al. (2002). ES can be used for any time series in which we can define events, such as singleneuron recordings, eptiform spikes in EEGs, heart beats, stock market crashes, or abrupt weather events, such as heavy rainfall events. However, ES is not limited to this definition of events. It could also be applied to time series which are pure event time series (e.g. heart beats). In principle, when dealing with signals of different characters, the events could be defined differently in each time series, since their common cause might manifest itself differently in each (Quiroga et al., 2002). ES has advantages over other time-delayed correlation techniques (e.g. Pearson lag correlation), as it allows us to study interrelations between series of non-Gaussian data or data with heavy tails, or to use a dynamical (non-constant) time delay (Tass et al., 1998; Stolbova et al., 2014). The latter refers to a time delay that is dynamically adjusted according to the two time series being compared, which allows for better adaptation to the region of interest. Furthermore, ES has been specifically designed to calculate non-linear linkages between time series. Various modifications of ES have been proposed, such as solving the problems of boundary effects and bias due to an infinite number of events (Stolbova et al., 2014; Malik et al., 2012; Rheinwalt et al., 2016).

The modified algorithm proposed by Stolbova et al. (2014), Malik et al. (2012), and Rheinwalt et al. (2016) works as follows: an event occurs in signals $x(t)$ and $y(t)$ at time $t_{l}^{x}$ and $t_{m}^{y}$, where $l=1,2,3,4, \ldots S_{x}, m=$
$1,2,3,4, \ldots S_{y}$, and $S_{x}$ and $S_{y}$ are the total number of events, respectively. In our study, we derive events from a more-orless continuous time series by selecting all time steps with values above a threshold ( $\alpha=95$ th percentile). These events in $x(t)$ and $y(t)$ are considered synchronized when they occur within a time lag $\pm \tau_{l m}^{x y}$ which is defined as follows:

$\tau_{l m}^{x y}=\min \left\{t_{l+1}^{x}-t_{l}^{x}, t_{l}^{x}-t_{l-1}^{x}, t_{m+1}^{y}-t_{m}^{y}, t_{m}^{y}-t_{m-1}^{y}\right\} / 2$.

This definition of the time lag helps to separate independent events, as it is the minimum time between two succeeding events. Then we count the number of times $C(x \mid y)$ an event occurs in $x(t)$ after it appears in $y(t)$ and vice versa $(C(y \mid x))$ :

$C(x \mid y)=\sum_{l=1}^{S_{x}} \sum_{m=1}^{S_{y} J_{x y}}$

and

$J_{x y}=\left\{\begin{array}{rlr}1 & \text { if } & 0<t_{l}^{x}-t_{m}^{y}<\tau_{l m}^{x y} \\ \frac{1}{2} & \text { if } & t_{l}^{x}=t_{m}^{y} \\ 0 & \text { else. } & \end{array}\right.$

$C(y \mid x)$ is calculated analogously but with exchanged $x$ and $y$. From these quantities we obtain the symmetric measure:

$Q_{x y}=\frac{C(x \mid y)+C(y \mid x)}{\sqrt{\left(S_{x}-2\right)\left(S_{y}-2\right)}}$.

$Q_{x y}$ is a measure of the strength of event synchronization between signals $x(t)$ and $y(t)$. It is normalized to $0 \leq Q_{x y} \leq$ 1 , with $Q_{x y}=1$ for perfect synchronization (coincidence of extreme events) between signals $x(t)$ and $y(t)$.

Recalling Eq. (6), the scale-wise approximation at different scales $0,1,2, \ldots, \lambda$ for any given time series $x(t)$ is given by $x_{\lambda}=A_{\lambda, q}$ where $x_{\lambda}$ represents the approximation coefficients of signal $x(\mathrm{t})$ at scale $\lambda$. Now, to determine the synchronization between any two time series $x(t)$ and $y(t)$ at multiple scales, the event synchronization is estimated between the scaled versions of $x(t)$ and $y(t)$ for different $\lambda$ resulting in multi-scale event synchronization (MSES). The normalized strength of MSES between signals $x(t)$ and $y(t)$ at scale $\lambda$ is then defined as

$Q^{x_{\lambda}, y_{\lambda}}=\frac{C\left(x_{\lambda} \mid y_{\lambda}\right)+C\left(y_{\lambda} \mid x_{\lambda}\right)}{\sqrt{\left(S_{x_{\lambda}}-2\right)\left(S_{y_{\lambda}}-2\right)}}$.

$Q^{x_{\lambda} y_{\lambda}}=1$ for perfect synchronization, and $Q^{x_{\lambda}, y_{\lambda}}=0$ suggests the absence of any synchronization at scale $\lambda$ between $x(t)$ and $y(t)$.

Figure 3 shows the stepwise methodology of multi-scale event synchronization. 
(a)
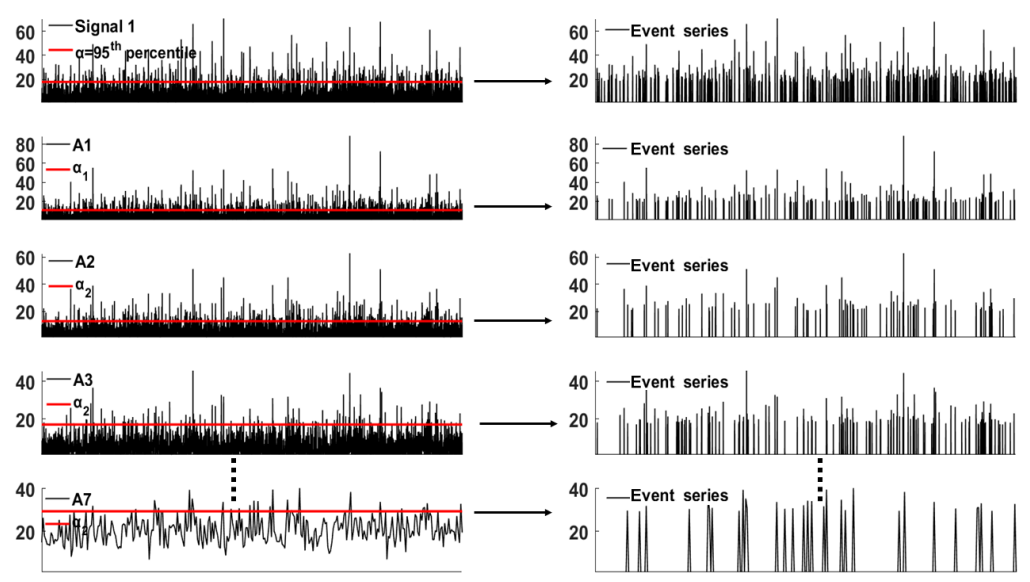

(b)
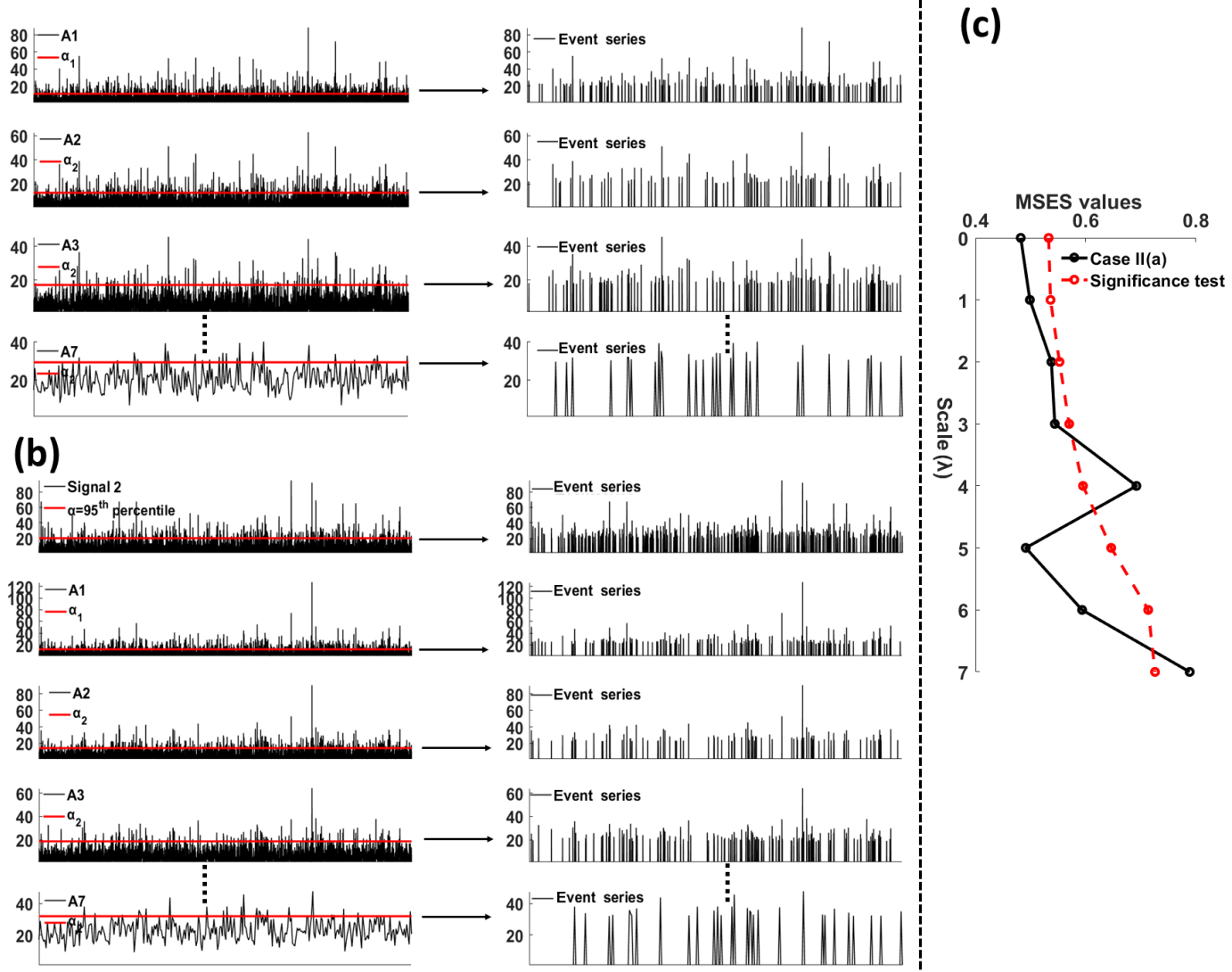

Figure 3. Multi-scale event synchronization (MSES) stepwise methodology. (a) Signal 1 and its decomposed component along with corresponding event series after applying the (95th percentile) threshold. (b) Same for signal 2. (c) Event synchronization values corresponding to each scale.

\subsection{Significance test for MSES}

To evaluate the statistical significance of ES values, a surrogate test will be used (Rheinwalt et al., 2016). We randomly reshuffle each time series 100 times (an arbitrary number). Reshuffling is done without replacement because estimating the expected number of simultaneous events in independent time series is equivalent to the combinatorial problem of sampling without replacement (Rheinwalt et al., 2016). Then, for each pair of time series, we calculate the MSES values for the different scales. At each scale, the empirical test distribution of the 100 MSES values for the reshuffled time series is compared to the MSES values of the original time series. Using a $1 \%$ significance level, we assume that synchronization cannot be explained by chance, if the MSES value at a certain scale of the original time series is larger than the 99th percentile of the test distribution.

\section{Data and study design to test MSES}

The proposed method is tested using synthetic and real-world data. The aim of these tests is to understand whether MSES is advantageous, compared to ES, in understanding the system interaction and the scale-emerging natural processes.

\subsection{Testing MSES with synthetic data}

Following the approach of Rathinasamy et al. (2014), Yan and Gao (2007), and $\mathrm{Hu}$ and $\mathrm{Si}$ (2016), we test MSES using a set of case studies including stationary and non-stationary synthetic data. The details of the case studies and the wavelet power spectra are given in Table 1 and Fig. 4, respectively.

Case I. A single synthetic stationary time series $(S)$ is generated and contaminated with two random white noise time series. Two sub-cases with different noise-signal ratios are investigated (Table 1). This case allows understanding of how the synchronization between two 

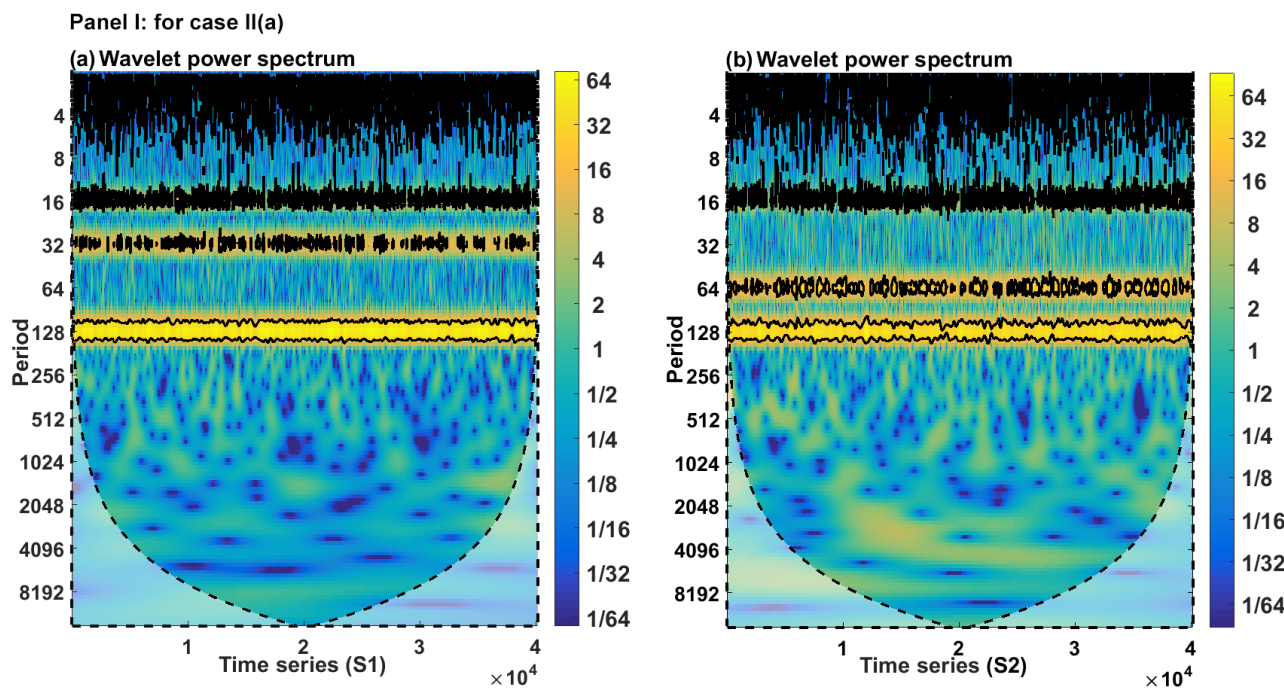

Panel II: for case II(b)
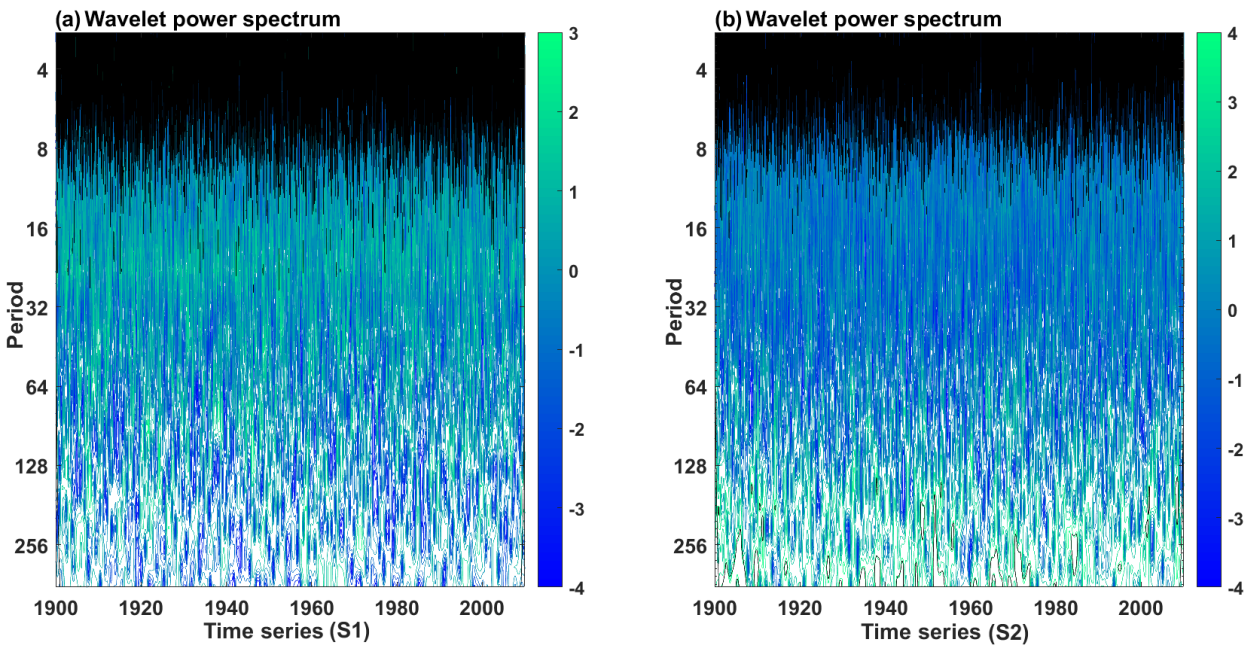

Panel III: for case III(a)
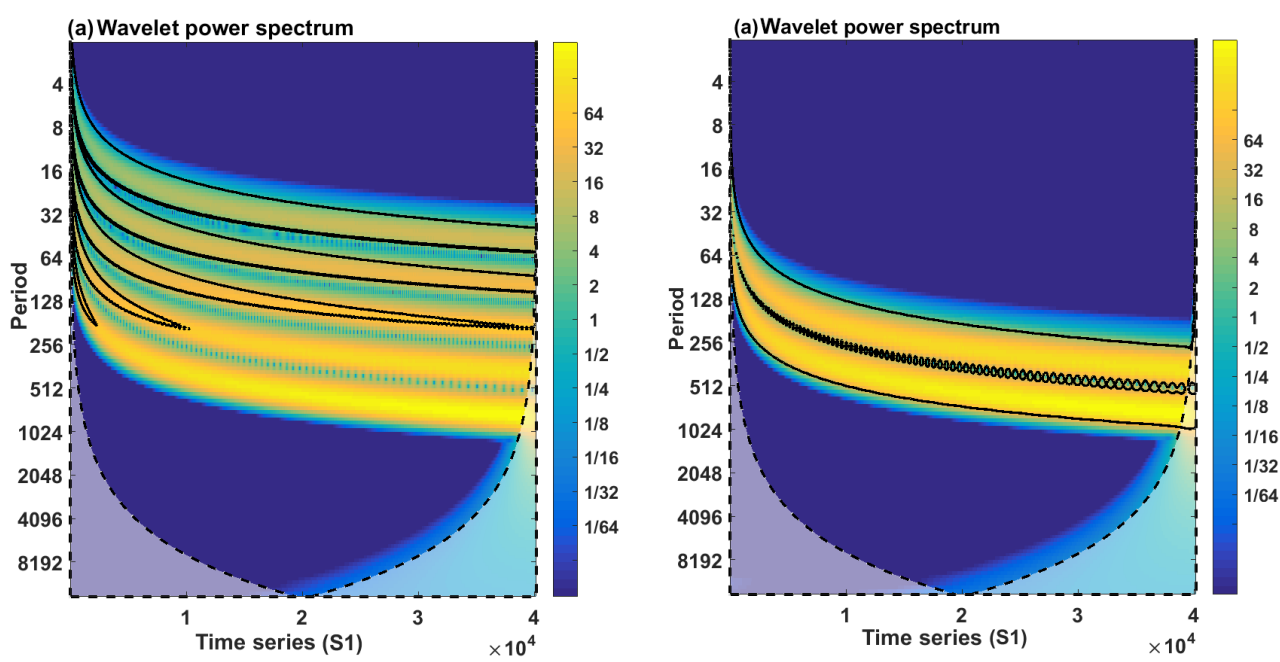

Figure 4. Wavelet power spectra (WPS) of the test signals (Table 1). Panel I: original signal S1 (left) and S2 (right), respectively, for case II(a); Panel II: original signal S1 (left) and S2 (right), respectively, for case II(b); Panel III: original signal S1 for case III(a); Panel IV: original signal S1 for case III(b). In all the panels, the y-axis represents the corresponding Fourier period $=2^{\lambda}$. 
series is affected by the presence of noise or highfrequency features. For climate variables such situations can emerge when two signals originate from the same parent source or mechanism (e.g. identical large-scale climatic mode, identical storm tracks) but get covered by high-frequency fluctuations arising from local features.

Case II(a). Here we generate two stationary signals consisting of partly shared long-term oscillations and autoregressive (AR1) noise $S_{t}$ (see Table 1). The long-term oscillations $y 1, y 2, y 3$, and $y 4$ have periods of 16,32 , 64, and 128 units, respectively (Fig. 4, Panel I). The purpose of case II(a) is to test the ability of MSES to identify synchronization in processes which originate from different parent sources or different mechanisms (e.g. two different climatic process, different storm tracks) but have some common features ( $y 1$ and $y 4)$ at coarser scales.

Case II(b) presents two signals (Fig. 4, Panel II) with no common features across all scales. Feature $y 2$ in signal S1 and feature $y 4$ in signal S2 represent a long-term oscillation of period 32 and 128 units, respectively. The idea is to investigate the possibility of overprediction of synchronization if we analyse at one scale only.

Case III. Here, MSES is tested using non-stationary signals (Fig. 4, Panel III and IV) generated as proposed by Yan and Gao (2007) and $\mathrm{Hu}$ and $\mathrm{Si}$ (2016). The signal encompasses five cosine waves ( $z 1$ to $z 5)$, whereas the square root of the location term results in a gradual change in frequency. Two combinations are generated of which case III(a) investigates the ability of MSES to deal with non-stationarity signals. Case III(b) examines the capability of MSES to capture processes emerging at lower scales (in this case at scales 5 and 6) in the presence of short-lived transient features. For both combinations, the signal is contaminated with white noise.

The time series of case III have features that are often found in climatic and geophysical data, where highfrequency, small-scale processes are superimposed on lowfrequency, coarse-scale processes (Hu and Si, 2016). Such structures are widespread in time series of seismic signals, turbulence, air temperature, precipitation, hydrologic fluxes, or the El Niño-Southern Oscillation. They can also be found in spatial data, e.g. in ocean waves, seafloor bathymetry, or land surface topography ( $\mathrm{Hu}$ and $\mathrm{Si}, 2016)$.

\subsection{Testing MSES with real-world data}

To test MSES with real-world data, we use precipitation data from stations in Germany (Fig. 5): 110 years of daily data, from 1 January 1901 to 31 December 2010, are available from various stations operated by the German Weather Ser-

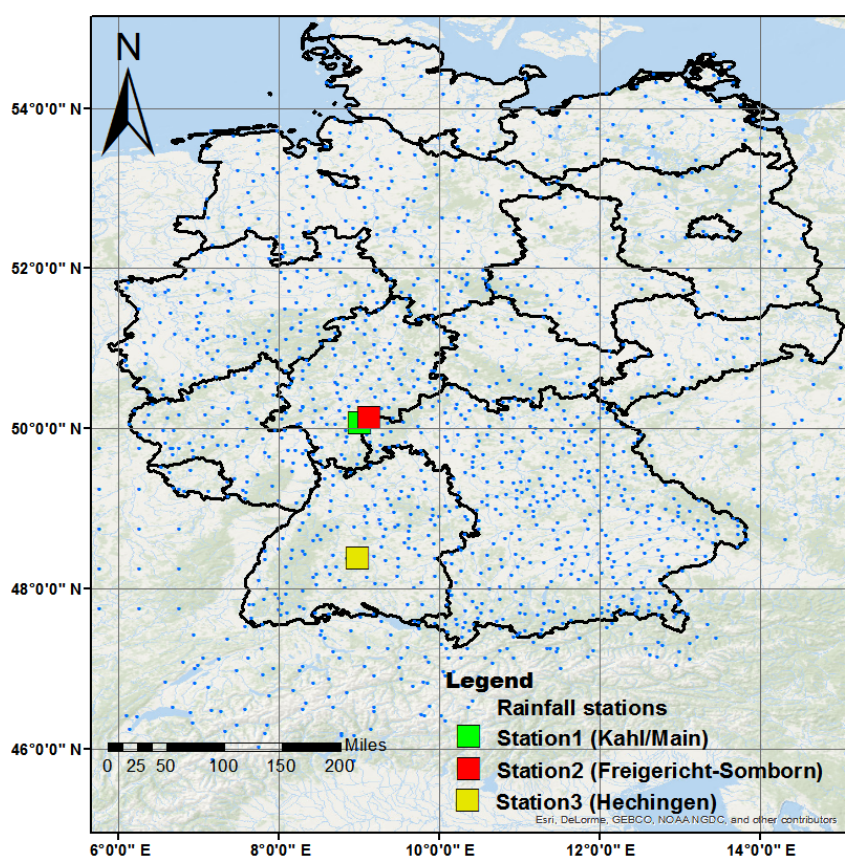

Figure 5. Geographical locations of rainfall stations considered in case study IV.

vice. Data processing and quality control were performed according to Österle et al. (2006).

Case IV. We use daily rainfall data from the three stations: Kahl/Main, Freigericht-Somborn, and Hechingen (station ID: 20009, 20208, and 25005). Considering Kahl/Main (station 1) as the reference station, the distance to the other two stations, Freigericht-Somborn (station 2) and Hechingen (station 3), are 14.88 and $185.62 \mathrm{~km}$, respectively (Fig. 5). Rainfall is a point process with large spatial and temporal discontinuities ranging from very weak to strong events within small temporal and spatial scales (Malik et al., 2012). This case explores the ability of MSES, in comparison to ES, to improve the understanding of synchronization given such time series features.

\section{Results}

To evaluate the synchronization between two signals, which can be expressed in terms of events, at multiple scales, we decompose the given time series up to a maximum scale beyond which there is no significant number event. The number of events at a scale is a function of the nature of the time series and also the length of the time series under consideration. In most cases it was found that the number of events was significantly reduced after seven or eight levels of decomposition. We use the Haar wavelet as this is one of the simplest but most basic mother wavelets. There are several other 
Table 1. Details of synthetic test cases.

\begin{tabular}{|c|c|c|c|}
\hline Case & Mathematical expression & Other details & References and figures \\
\hline I(a) & $\begin{array}{l}\text { Sinusoidal stationary signal } \\
\mathrm{S} 1=S+\text { Strong noise }_{1}\end{array}$ & $\begin{array}{l}S=\sin ((2 \pi t) / 50)+\cos ((2 \pi t) / 60) \\
\frac{\text { Noise }}{\text { signal }} \sim 2.8\end{array}$ & $\begin{array}{l}\text { Rathinasamy et } \\
\text { al. (2014) }\end{array}$ \\
\hline $\mathrm{I}(\mathrm{b})$ & $\begin{array}{l}\text { Sinusoidal stationary signal } \\
\mathrm{S} 2=S+\text { Weaknoise }_{2}\end{array}$ & $\frac{\text { Noise }_{2}}{\text { signal }} \sim 5$ & $\begin{array}{l}\text { Rathinasamy et } \\
\text { al. (2014) }\end{array}$ \\
\hline $\mathrm{II}(\mathrm{a})$ & $\begin{array}{l}\text { Stationary signal (S1 and } \mathrm{S} 2) \\
\mathrm{S} 1=S_{t_{1}}+y 1+y 2+y 4 \\
\mathrm{~S} 2=S_{t_{2}}+y 1+y 3+y 4\end{array}$ & $\begin{array}{l}\text { Two AR1 processes } S_{t}=\varnothing S_{t-1}+\epsilon_{t} \\
\varepsilon_{t}=\text { uncorrelated random noise } \\
\text { Parameter }\left\{\varnothing_{1}=0.60 ; \varnothing_{2}=.70\right\}\end{array}$ & $\begin{array}{l}\text { Yan and Gao (2007), } \\
\text { Hu and Si (2016) } \\
\text { Fig. 3: Panel I }\end{array}$ \\
\hline $\mathrm{II}(\mathrm{b})$ & $\begin{array}{l}\text { Stationary dataset (S1 and } \mathrm{S} 2) \\
\mathrm{S} 1=y 2+S_{t 1} \\
\mathrm{~S} 2=y 4+S_{t_{2}}\end{array}$ & $\begin{array}{l}y 1=\sin \left(\frac{2 \pi t}{16}\right) ; y 2=\sin \left(\frac{2 \pi t}{32}\right) \\
y 3=\sin \left(\frac{2 \pi t}{64}\right) ; y 4=\sin \left(\frac{2 \pi t}{128}\right) \\
\text { where } t=1,2,3, \ldots 40177\end{array}$ & $\begin{array}{l}\text { Yan and Gao (2007), } \\
\text { Hu and Si (2016) } \\
\text { Fig. 3: Panel II }\end{array}$ \\
\hline $\mathrm{III}(\mathrm{a})$ & $\begin{array}{l}\text { Non-stationary dataset } \\
\mathrm{S} 1=z 1+z 2+z 3+z 4+z 5 \\
\mathrm{~S} 2=\mathrm{S} 1+\text { random noise } \\
\text { (uncorrelated) } \\
\text { noise } \sim 2.781 ; \\
\text { signal } \sim \\
\text { where } t=1,2,3, \ldots 40177 .\end{array}$ & $\begin{array}{l}Z 1=\cos \left(500 \pi\left(\frac{t}{1000}\right)^{0.5}\right), \\
Z 2=\cos \left(250 \pi\left(\frac{t}{1000}\right)^{0.5}\right), \\
Z 3=\cos \left(125 \pi\left(\frac{t}{1000}\right)^{0.5}\right), \\
Z 4=\cos \left(62.5 \pi\left(\frac{t}{1000}\right)^{0.5}\right), \\
Z 5=\cos \left(31.25 \pi\left(\frac{t}{1000}\right)^{0.5}\right) .\end{array}$ & $\begin{array}{l}\text { Yan and Gao (2007), } \\
\text { Hu and Si (2016) } \\
\text { Fig. 3: Panel III }\end{array}$ \\
\hline $\mathrm{III}(\mathrm{b})$ & $\begin{array}{l}\text { Non-stationary dataset } \\
\mathrm{S} 1=z 4+z 5 \\
\mathrm{~S} 2=\mathrm{S} 1+\text { random noise } \\
\text { (uncorrelated) } \\
\text { noise } \sim 21.5664 ; \\
\text { signal } t=1,2,3, \ldots 40177 .\end{array}$ & $\begin{array}{l}Z 4=\cos \left(62.5 \pi\left(\frac{t}{1000}\right)^{0.5}\right), \\
Z 5=\cos \left(31.25 \pi\left(\frac{t}{1000}\right)^{0.5}\right) .\end{array}$ & $\begin{array}{l}\text { Yan and Gao (2007), } \\
\text { Hu and Si (2016) } \\
\text { Fig. 3: Panel IV }\end{array}$ \\
\hline
\end{tabular}

mother wavelets which could be used for wavelet decomposition; however, it has been demonstrated that the choice of the mother wavelets does not affect the results to a great extent for rainfall (Rathinasamy et al., 2014).

In case I(a) the noise-signal ratio is quite high in the range of 2.7-3 (Table 1), such that the effect of the noise is felt up to scale 7 (Fig. 6). Although both signals stem from the same parent source and hence ideally they should possess perfect synchronization $(\mathrm{ES} \sim 1)$ at all scales, the $\mathrm{ES}$ value at the observational scale $(\lambda=0)$ is moderate $(\sim 0.7)$, leading to the interpretation that both signals are only weakly synchronized. In contrast, the proposed MSES approach is able to capture the underlying features (which were hidden in the original signal) at higher scales $(\lambda \geq 1)$ by approaching ES values of 1 , indicating the actual synchronization between these signals. At the scale $\lambda=0$ the ES measure is lower because of the heavy noise covering the underlying information. Considering higher scales, the effect of noise is removed through wavelet decomposition, allowing for a more reliable identification of the actual underlying synchronization between the signals. Interestingly, the slight decrease in the ES values at a high scale $(\lambda \geq 7)$ (Fig. 6) might indicate that the essential feature that is responsible for the synchronization at that scale gets removed in the form of a detail component (Fig. 2). If features are present at a particular scale $\lambda$ and when we go up to the next scale $(\lambda+1)$, those features get removed in the form of the details and essentially the synchronization is lost at the scale $\lambda+1$.

While repeating the same analysis but with a lower noisesignal ratio (i.e. case $\mathrm{I}(\mathrm{b})$ ), we find that the effect of noise is almost completely removed after $(\lambda>3)$ and the MSES values remain unaltered because of the same signal structure (Fig. 6). These findings confirm that the MSES approach is able to capture the synchronization in the presence of noise.

The significance test (Sect. 2.4) underlines the high level of synchronization as indicated by the quite high ES values (Fig. 6). Based on this example we find that the MSES analysis captures the synchronization at multiple scales.

Case II(a) presents a system where synchronization between two signals exists at a common long-term frequency ( $y 1$ and $y 4)$. This is particularly relevant in studying the rainfall processes of two different regions, which are governed by different local climatic processes but similar long-term oscillations such as ENSO cycles. The MSES values $(\lambda=0$ to 7$)$ are smaller than the confidence level, except for scales 4 and 7 (Fig. 7a). The synchronization emerging at scale $4(\lambda=4)$ 


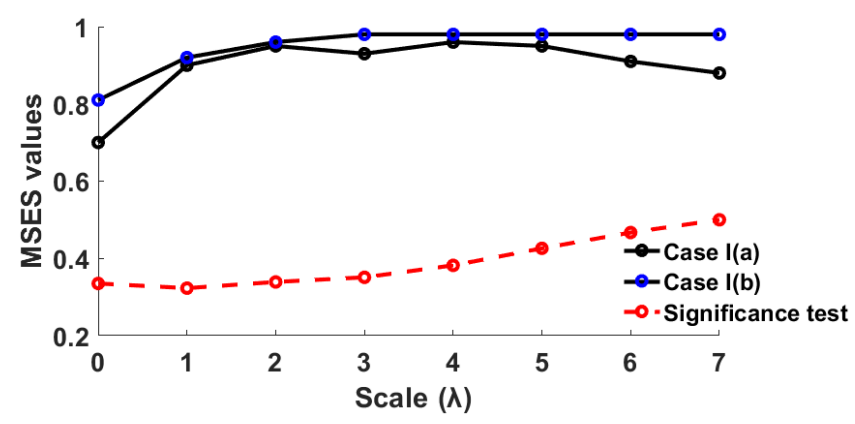

Figure 6. MSES values for case I(a) and case I(b), including significance test values for the significance level of $1 \%$. The value at scale 0 is equal to the single-scale ES analysis.

and scale $7(\lambda=7)$ corresponds to features present at those scales shown in the wavelet power spectrum (Fig. 5, Panel I). The thick contour in the WPS indicates the presence of significant features (at the $5 \%$ significance level) corresponding to $y 1, y 2, y 3$, and $y 4$ (Table 1). In the same figure, the dashed curve represents the cone of influence (COI) of the wavelet analysis. Outside of this region edge effects become more influential. Any peak falling outside the COI has presumably been reduced in magnitude due to zero padding necessary to deal with the finite length of the time series. To test the statistical significance of WPS, a background Fourier spectrum is chosen (Addison, 2005; Agarwal et al., 2016a, b).

For case II(b), we would expect that the ES value should be zero or nonsignificant at scale $\lambda=0$. However, we find that the synchronization between $\mathrm{S} 1$ and $\mathrm{S} 2$ at scale $\lambda=0$ is significant (Fig. 7b), although there is no common feature by construction (Fig. 3, Panel II).

Interestingly, the MSES does not find significant synchronization at any scale $(\lambda>0)$. Moreover, the MSES values become zero after scale 4 because signals $S 1$ and S2 have no common feature beyond these scales.

As seen clearly, the ES at only one scale overpredicts the actual synchronicity between the two series. This behaviour may be due to the integrated effect of all scales, and hence some spurious synchronization (although rather small but still significant) is indicated.

Case III(a) is used as an analogue of dynamics and features of natural processes (Table 1). Its WPS (Fig. 4, Panel III) shows non-stationary, time-dependent features at higher scales $2 \leq \lambda \leq 6$. ES values at lower scales $\lambda \leq 1$ are below the significance level, revealing that the two signals are not synchronized (Fig. 8a). The ES for the signal components of the larger timescales reveals significant synchronization up to scale 6 , which is expected because of the common features (scale 2 to scale 6) in S1 and S2. After scale $\lambda=6$, the MSES value drops below the significance level as the features responsible for synchronization are removed in the form of the details component during decomposition. Results from this case show the wavelet's ability in capturing the underlying multiple non-stationarities that are common in both the time series which otherwise go unnoticed using ES at the observation scale.

The similar case III(b) is used to investigate the behaviour of MSES in a scale-emerging process in a non-stationary regime (Table 1). As the wavelet spectrum of the signal reveals, only features at scales 5 and 6 are present (Fig. 4, Panel IV). The corresponding MSES values are significant only at those scales (Fig. 8b), revealing the synchronization at scales 5 and 6 . This case illustrates that MSES reveals only the relevant timescales and does not mix them with the observation scale. In reality, there may be situations where the causative events act only at certain timescales and remain unconnected at other timescales. Under such situations MSES is useful for unravelling the relevant scale-emerging relationships.

After testing the efficacy of the proposed MSES approach by using some prototypical situations, we apply the approach to real observed rainfall data (case IV). We find significant ES values between station 1 and station 2 at the scales $\lambda=1$, 5 , and 7 (Fig. 9a) by tracking the features present in the WPS (Fig. 9c, d, and e). The significant ES value at the observational scale $(\lambda=0)$ might be due to the integrated effect of features present at coarser scales $(\lambda=1,5$, and 7$)$. In order to emphasize the features present in the data, we use the global wavelet spectrum (Fig. 9f, g, and h) which is defined as the time average of the WPS (Agarwal et al., 2016a, b; Mallat, 1989).

Applying ES in the traditional way, i.e. analysing only at scale 0 , we find synchronization. However, only when we consider multiple scales are we able to find that the synchronization is the result of high- and low-frequency components present at scales 1,5 , and 7 .

For station 1 and station 3 synchronization is significant at scale $7(\lambda=7)$ (Fig. 9b). However, evaluating the ES in the traditional way (i.e. $\lambda=0$ ) leads to the conclusion that both stations are not significantly synchronized. Here, MSES plays a critical role in identifying synchronization at specific temporal scales. Hence, MSES provides further insights into the process, such as low-frequency features that are present and the dominating scales causing the significant synchronization at scale 0 .

The results for the real-world case study suggest that proximity of stations (station 1 and station 2) does not necessarily indicate synchronization at all scales. For stations 1 and 3 , which are comparatively far from each other, we find insignificant synchronization at the observational scale. However, considering the scales separately, MSES detects significant synchronization at scale 7 as both stations might be sharing some common climatic cycle at this scale. 

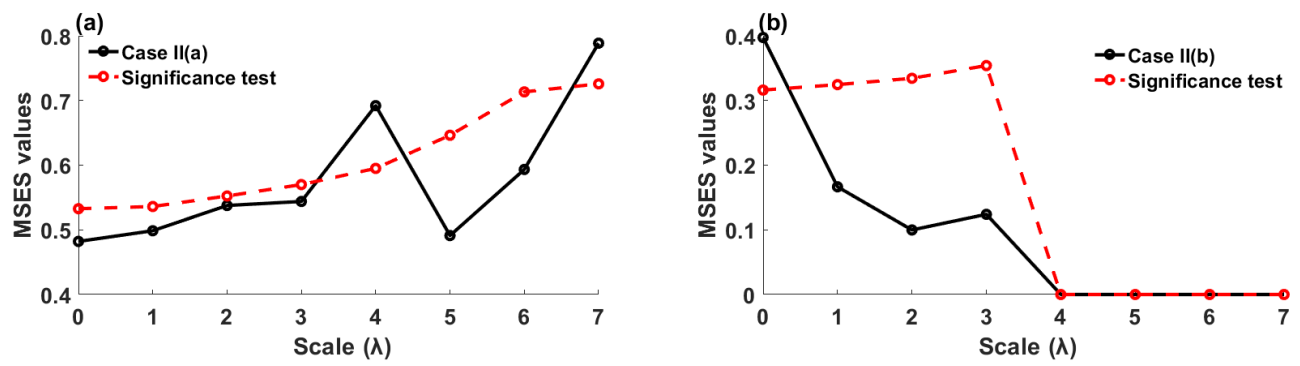

Figure 7. (a, b) MSES and significance level (1\%) values at different scales for cases II(a) and II(b). The value at scale 0 is equal to the single-scale ES analysis.
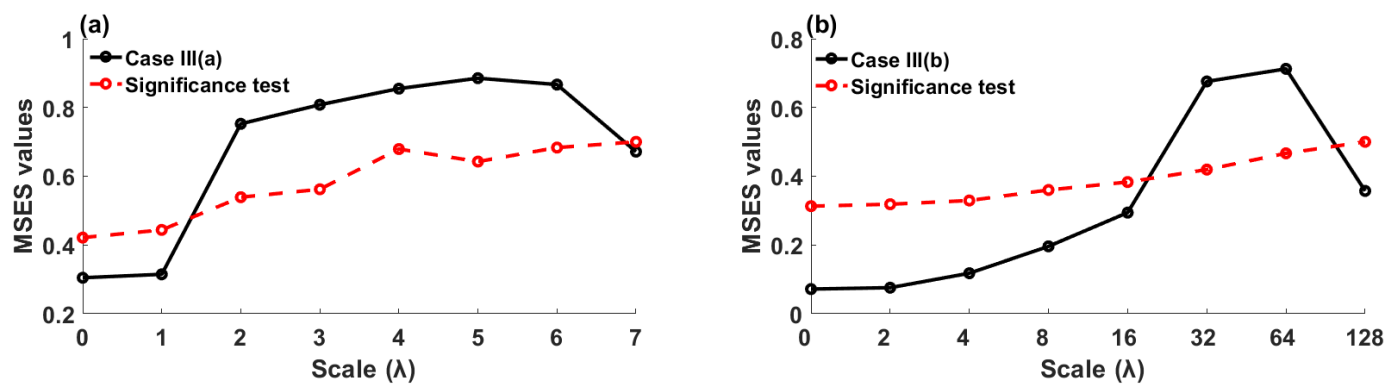

Figure 8. (a, b) MSES values and significance level (1\%) at different scales for cases III(a) and III(b). The value at scale 0 is equal to the single-scale ES analysis.

\section{Discussion}

We have compared our novel MSES method with the traditional ES approach by systematically applying both methods to a range of prototypical situations. For test cases I and II we find that the ES value at the observation scale is influenced by noise, thereby reducing the ES values of two actually synchronized time series. When using MSES, the synchronization between the two time series can be much better detected even in the presence of strong noise. Another important aspect related to the analysis of these cases is that MSES has the ability to unravel synchronization between two stationary systems at timescales which are not obvious at the observation scale (scale-emerging processes). From these observations, it becomes clear that (i) event synchronization only at a single scale of reference is less robust, and (ii) the dependency measure of two given processes based on ES changes with the timescale depending on the features present in these processes.

Case study III illustrates that for a non-stationary system with synchronization changing over temporal scales, the single-scale ES is not robust. In contrast, MSES uncovers the underlying synchronization clearly. MSES is able to track the scale-emerging processes, scale of dominance in the process, and features present.

The real-world case study IV shows that the synchronization between climate time series can differ with temporal scales. The strength of synchronization as a function of tem- poral scale might result from different dynamics of the underlying processes. MSES has the ability to uncover the scale of dominance in the natural process.

Our series of test cases confirms the importance of applying a multi-scale view in order to investigate the relationship between processes that exist at different timescales. We suggest that investigating synchronization just at a single, i.e. observational, scale could give limited insight. The proposed extension offers the possibility of deciphering synchronization at different timescales, which is important in the case of climate systems where feedbacks and synchronization occur only at certain timescales and are absent at other scales.

\section{Conclusions}

We have proposed a novel method which combines wavelet transforms with event synchronization, thereby allowing us to investigate the synchronization between event time series at a range of temporal scales. Using a range of prototypical situations and a real-world case study, we have shown that the proposed methodology is superior compared to the traditional event synchronization method. MSES is able to provide more insight into the interaction between the analysed time series. Also, the effect of noise and local disturbance can be reduced to a greater extent and the underlying interrelationship becomes more prominent. This is attributed to the fact that wavelet decomposition provides a multi-resolution representation which helps to improve the estimation of syn- 

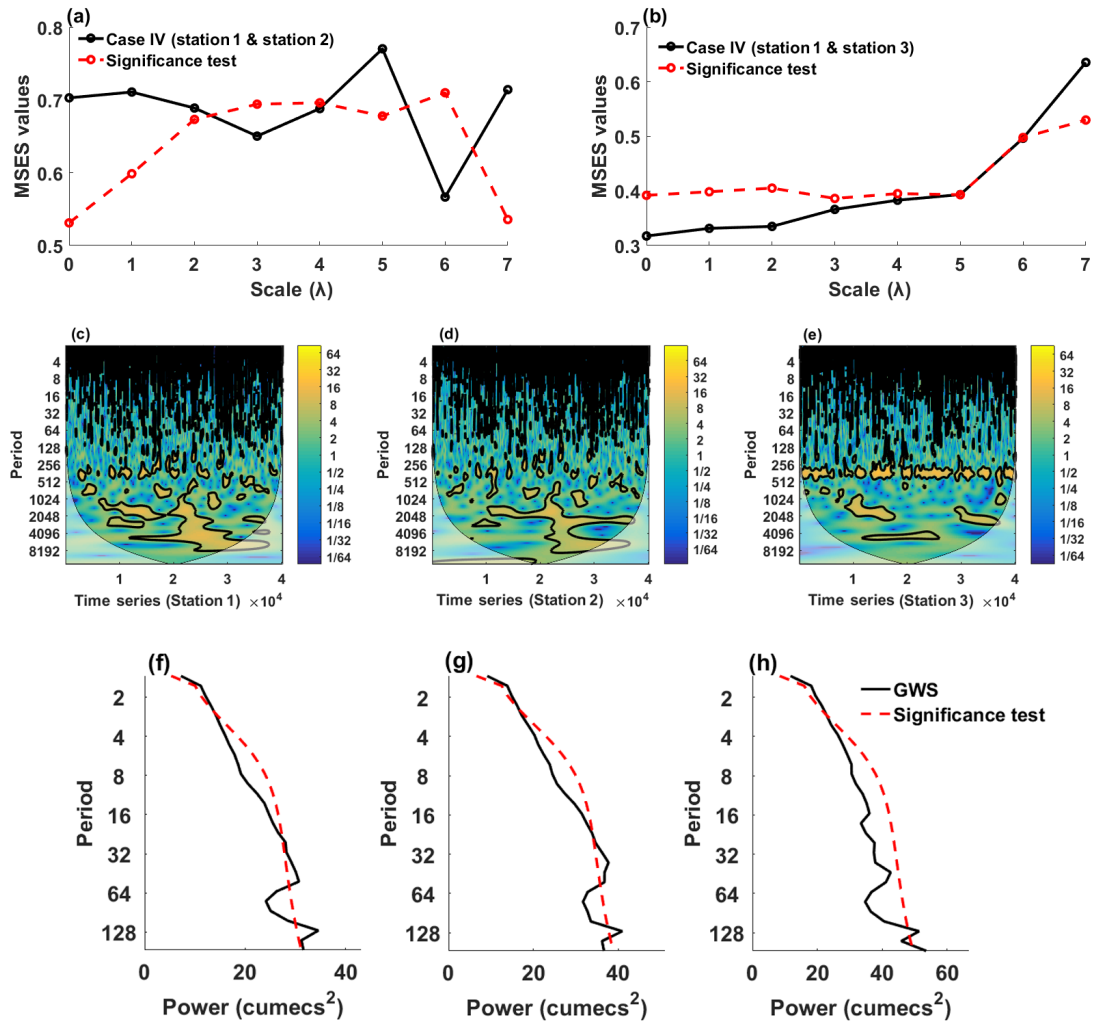

Figure 9. (a, b) MSES and significance level (1\%) values at various scales for stations 1 and 2 and stations 1 and 3, respectively; (c, d, e) WPS of precipitation of stations 1 (c), 2 (d), and 3 (e) (station ID: 20009, 20208, 25005), respectively; (f, g, h) global wavelet spectrum of the same stations. In (c)-(h) the $y$-axis represents the corresponding Fourier period $=2^{\lambda}$.

chronization. Another advantage of the proposed approach is its ability to deal with non-stationarity. Wavelets being made on local bases can pick up the non-stationary, transient features of a system, thereby improving the estimation of ES. Finally, it can be concluded that the proposed method is more robust and reliable than the traditional event synchronization in estimating the relationship between two processes.

Data availability. The authors used Germany's precipitation data which is maintained and provided by German Weather Service. The data is publicly accessible at https://opendata.dwd.de/. Further, preprocessing of the data was done by Potsdam Institute for Climate Impact Research (Conradt et al., 2012; Oesterle, 2001).

Competing interests. The authors declare that they have no conflict of interest.

Acknowledgements. This research was funded by the Deutsche Forschungsgemeinschaft (DFG) (GRK 2043/1) within graduate research training group Natural risk in a changing world (NatRiskChange) at the University of Potsdam (http://www.uni-potsdam.de/natriskchange) and RSF support (sup- port by the Russian Science Foundation (grant no. 16-12-10198)) The third author acknowledges the research funding from the Inspire Faculty Award, Department of Science and Technology, India, for carrying out the research. Also, we gratefully acknowledge the provision of precipitation data by the German Weather Service.

Edited by: Stéphane Vannitsem

Reviewed by: two anonymous referees

\section{References}

Addison, P. S.: Wavelet transforms and the ECG: a review, Physiol. Meas., 26, R155, 2005.

Agarwal, A., Maheswaran, R., Sehgal, V., Khosa, R., Sivakumar, B., and Bernhofer, C.: Hydrologic regionalization using waveletbased multiscale entropy method, J. Hydrol., 538, 22-32, 2016a.

Agarwal, A., Maheswaran, R., Kurths, J., and Khosa, R.: Wavelet Spectrum and Self-Organizing Maps-Based Approach for Hydrologic Regionalization - a Case Study in the Western United States, Water Resour. Manag., 30, 4399-4413, https://doi.org/10.1007/s11269-016-1428-1, 2016 b.

Arnhold, J., Grassberger, P., Lehnertz, K., and Elger, C. E.: A robust method for detecting interdependences: application to intracranially recorded EEG, Physica D, 134, 419-430, 1999. 
Barrat, A., Barthelemy, M., and Vespignani, A.: Dynamical processes on complex networks, Cambridge university press, 2008.

Callahan, D., Kennedy, K., and Subhlok, J.: Analysis of event synchronization in a parallel programming tool, ACM SIGPLAN Notices, 21-30, 1990.

Conradt, T., Koch, H., Hattermann, F. F., and Wechsung, F.: Precipitation or evapotranspiration? Bayesian analysis of potential error sources in the simulation of sub-basin discharges in the Czech Elbe River basin, Reg. Environ. Change, 12, 649-661, https://doi.org/10.1007/s10113-012-0280-y, 2012.

Donner, R. V., Zou, Y., Donges, J. F., Marwan, N., and Kurths, J.: Recurrence networks - a novel paradigm for nonlinear time series analysis, New J. Phys., 12, 033025, https://doi.org/10.1088/1367-2630/12/3/033025, 2010.

Eroglu, D., McRobie, F. H., Ozken, I., Stemler, T., Wyrwoll, K.-H., Breitenbach, S. F., Marwan, N., and Kurths, J.: See-saw relationship of the Holocene East Asian-Australian summer monsoon, Nat. Commun., 7, 12929, https://doi.org/10.1038/ncomms12929, 2016.

Herlau, T., Mørup, M., Schmidt, M. N., and Hansen, L. K.: Modelling dense relational data, Machine Learning for Signal Processing (MLSP), IEEE International Workshop, 1-6, 2012,

$\mathrm{Hu}, \mathrm{W}$. and Si, B. C.: Technical note: Multiple wavelet coherence for untangling scale-specific and localized multivariate relationships in geosciences, Hydrol. Earth Syst. Sci., 20, 3183-3191, https://doi.org/10.5194/hess-20-3183-2016, 2016.

Krause, C. M., Lang, A. H., Laine, M., Kuusisto, M., and Pörn, B.: Event-related. EEG desynchronization and synchronization during an auditory memory task, Electroen. Clin. Neuro., 98, 319326, 1996.

Le Van Quyen, M., Martinerie, J., Adam, C., and Varela, F. J.: Nonlinear analyses of interictal EEG map the brain interdependences in human focal epilepsy, Physica D, 127, 250-266, 1999.

Liang, Z., Ren, Y., Yan, J., Li, D., Voss, L. J., Sleigh, J. W., and Li, $\mathrm{X}$.: A comparison of different synchronization measures in electroencephalogram during propofol anesthesia, J. Clin. Monitor. Comp., 30, 451-466, 2016.

Malik, N., Bookhagen, B., Marwan, N., and Kurths, J.: Analysis of spatial and temporal extreme monsoonal rainfall over South Asia using complex networks, Clim. Dynam., 39, 971-987, 2012.

Mallat, S. G.: A theory for multiresolution signal decomposition: the wavelet representation, IEEE T. Pattern Anal., 11, 674-693, 1989.

Maraun, D. and Kurths, J.: Epochs of phase coherence between El Nino/Southern Oscillation and Indian monsoon, Geophys. Res. Lett., 32, https://doi.org/10.1029/2005GL023225, 2005.

Marwan, N., Romano, M. C., Thiel, M., and Kurths, J.: Recurrence plots for the analysis of complex systems, Phys. Rep., 438, $237-$ 329, 2007.

Miritello, G., Moro, E., Lara, R., Martínez-López, R., Belchamber, J., Roberts, S. G., and Dunbar, R. I.: Time as a limited resource: Communication strategy in mobile phone networks, Soc. Networks, 35, 89-95, 2013.

Mokhov, I. I., Smirnov, D. A., Nakonechny, P. I., Kozlenko, S. S., Seleznev, E. P., and Kurths, J.: Alternating mutual influence of El-Niño/Southern Oscillation and Indian monsoon, Geophys. Res. Lett., 38, 47-56, https://doi.org/10.1134/S0001433812010082, 2011.
O'Connor, J. M., Pretorius, P. H., Johnson, K., and King, M. A.: A method to synchronize signals from multiple patient monitoring devices through a single input channel for inclusion in list-mode acquisitions, Med. Phys., 40, 122502, https://doi.org/10.1118/1.4828844, 2013.

Oesterle, H.: Reconstruction of daily global radiation for past years for use in agricultural models, Phys. Chem. Earth Part B, 26, 253-256, https://doi.org/10.1016/S1464-1909(00)002483, 2001.

Österle, H., Werner, P., and Gerstengarbe, F.: Qualitätsprüfung, Ergänzung und Homogenisierung der täglichen Datenreihen in Deutschland, 1951-2003: ein neuer Datensatz, 7. Deutsche Klimatagung, Klimatrends: Vergangenheit und Zukunft, 9.-11. Oktober 2006, München, 2006.

Perra, N., Gonçalves, B., Pastor-Satorras, R., and Vespignani, A.: Activity driven modeling of time varying networks, arXiv preprint arXiv:1203.5351, 2012.

Pfurtscheller, G. and Da Silva, F. L.: Event-related EEG/MEG synchronization and desynchronization: basic principles, Clin. Neurophysiol., 110, 1842-1857, 1999.

Quiroga, R. Q., Arnhold, J., and Grassberger, P.: Learning driverresponse relationships from synchronization patterns, Phys. Rev. E, 61, 5142, https://doi.org/10.1103/PhysRevE.61.5142, 2000.

Quiroga, R. Q., Kraskov, A., Kreuz, T., and Grassberger, P.: Performance of different synchronization measures in real data: a case study on electroencephalographic signals, Phys. Rev. E, 65, 041903, https://doi.org/10.1103/PhysRevE.65.041903, 2002.

Rathinasamy, M., Khosa, R., Adamowski, J., Partheepan, G., Anand, J., and Narsimlu, B.: Wavelet-based multiscale performance analysis: An approach to assess and improve hydrological models, Water Resour. Res., 50, 9721-9737, 2014.

Rheinwalt, A., Boers, N., Marwan, N., Kurths, J., Hoffmann, P., Gerstengarbe, F.-W., and Werner, P.: Non-linear time series analysis of precipitation events using regional climate networks for Germany, Clim. Dynam., 46, 1065-1074, 2016.

Rial, J. A.: Synchronization of polar climate variability over the last ice age: in search of simple rules at the heart of climate's complexity, Am. J. Sci., 312, 417-448, 2012.

Rosenblum, M. G., Pikovsky, A. S., and Kurths, J.: From phase to lag synchronization in coupled chaotic oscillators, Phys. Rev. Lett., 78, 4193-4196, https://doi.org/10.1103/PhysRevLett.78.4193, 1997.

Schiff, S. J., So, P., Chang, T., Burke, R. E., and Sauer, T.: Detecting dynamical interdependence and generalized synchrony through mutual prediction in a neural ensemble, Phys. Rev. E, 54, 67086724, https://doi.org/10.1103/PhysRevE.54.6708, 1996.

Schmocker-Fackel, P. and Naef, F.: Changes in flood frequencies in Switzerland since 1500, Hydrol. Earth Syst. Sci., 14, 1581-1594, https://doi.org/10.5194/hess-14-1581-2010, 2010.

Steinhaeuser, K., Ganguly, A. R., and Chawla, N. V.: Multivariate and multiscale dependence in the global climate system revealed through complex networks, Clim. Dynam., 39, 889-895, 2012.

Stolbova, V., Martin, P., Bookhagen, B., Marwan, N., and Kurths, J.: Topology and seasonal evolution of the network of extreme precipitation over the Indian subcontinent and Sri Lanka, Nonlinear Proc. Geoph., 21, 901-917, 2014.

Tass, P., Rosenblum, M. G., Weule, J., Kurths, J., Pikovsky, A., Volkmann, J., Schnitzler, A., and Freund, H.-J.: Detection of $\mathrm{n}$ : $\mathrm{m}$ phase locking from noisy data: application to 
magnetoencephalography, Phys. Rev. Lett., 81, 3291-3294, https://doi.org/10.1103/PhysRevLett.81.3291, 1998.

Tsui, C. Y.: A Multiscale Analysis Method and its Application to Mesoscale Rainfall System, Universität zu Köln, 2015.
Yan, R. and Gao, R. X.: A tour of the tour of the Hilbert-Huang transform: an empirical tool for signal analysis, IEEE Instru. Meas. Mag., 10, 40-45, 2007. 